\title{
ADAPTIVE TRANSCODING PROXY ARCHITECTURE FOR VIDEO STREAMING IN MOBILE NETWORKS
}

\author{
Matthias Dick, Jens Brandt, Verena Kahmann, Lars Wolf \\ Institute of Operating Systems and Computer Networks \\ Technische Universität Braunschweig \\ Mühlenpfordtstr. 23 \\ 38106 Braunschweig, Germany \\ Email: \{dick, brandt, kahmann, wolf $\} @$ ibr.cs.tu-bs.de
}

\begin{abstract}
Wireless video streaming is an area of extensive research due to the error-prone nature of the radio channel with variations in throughput and delay. In mobile networks, handovers may lead to further service degradation. Therefore, adaptation has to be applied in order to cope with these challenges. This paper introduces a new two-level proxy architecture which addresses the aforementioned issues. The first level proxy provides media adaptation to the requirements of its clients by using transcoding techniques and thereby supports many different mobile devices. The second level proxy is supposed to perform adaptation to the radio channel by using FGS-coding as well as unequal error protection. We also discuss handover QoS issues and approaches for signaling with service composition. Our two-level design is highly flexible and provides efficient, scalable and low-latency mobile streaming video. Besides, it provides better core network resource utilization while solving wireless channel problems where they occur.
\end{abstract}

\section{INTRODUCTION}

Video streaming over the Internet has attracted a large interest in commercial as well as in research areas. However, the physical characteristics of wireless networks like shadowing and multi-path fading, co-channel interferences, dynamic topologies and mobility lead to high bit error rate (BER) fluctuations and make robust wireless video streaming a challenging task. Furthermore, the available bit-rate in wireless communication channels is also highly limited. Therefore, transmission efficiency is an important factor in a wireless communication system. And finally, energy consumption of mobile devices should be taken into account, because of limited battery resources.

In order to achieve a robust data transmission, proactive error control techniques like forward error correction (FEC) and reactive techniques like automatic repeat request (ARQ) have been considered. However, as we show in section 2, none of these approaches can fulfill all quality criteria by itself. Instead, applying a scalable coding scheme to achieve efficiency in combination with unequal error protection for error resilience is reasonable. We also use feedback about the channel state to adapt the coding scale as well as the level of error protection. Thus, a robust and efficient transmission scheme that adapts well to varying channel characteristics is provided.

In addition to the aforementioned problems of the radio channel another problem of wireless networks is the high level of heterogeneity of mobile devices. Due to limited resources such as processing power, display resolution or supported media formats mobile devices often cannot comply with the requirements of high quality media streams. To overcome this problem each stream can be adapted to the requirements of the requesting client. One promising approach to achieve this is the use of transcoding techniques on intermediate proxies, which are integrated into the data path of the stream. This is advantageous, since content providers not necessarily have to provide different versions of a stream. One high-quality version is sufficient because it can be transcoded individually to the requirements of mobile devices.

This leads to the combination of both transcoding and adaptation techniques using a two-level proxy architecture. In our RASMUS (Robust Adaptive Service for MUltimedia Streaming) architecture, we use a Transcoding Proxy in the fixed network, which applies on-demand transcoding of media according to the needs of client devices and bandwidth restrictions, and a channel adaptation component, which uses both source and channel coding techniques to cope with the wireless channel characteristics. The latter also deals with error protection and thus provides a robust service.

The benefits of using a two-level architecture are that error protection can be done where errors occur (by use of the channel adaptation component) and bandwidth resources in the mobile core network are utilized carefully (by use of the transcoding proxy). With our service composition signaling approach, location of the components is delegated to a Service Manager entity, thus a mobile device does not have to care about the actual adaptive service setup.

The rest of this paper is organized as follows. Section 2 provides a review of the related work. In section 3 , we introduce our new two-level proxy architecture and present the Transcoding Proxy. Section 4 describes the Channel Adaptation Proxy. Section 5 deals with signaling aspects of our architecture. Finally, we summarize the conclusions and outline our future work.

\section{RELATED WORK}

Classical approaches to handle transmission errors have been a research topic for many years, and still are elaborated further due to the needs of robust wireless transmission.

Among reactive techniques, ARQ is most well known because of its determinateness and the ability to combine both error handling and flow control. However, ARQ requires a feedback channel and introduces additional delay and jitter 
which is undesirable for real-time applications. To avoid such delay, Error Tracking (ET) [1] has been invented, where lost packets, i. e. regions of a frame, are indicated by feedback. The idea then is to prevent error propagation to subsequent predictive-coded frames by switching to intra-frame coding for these corrupted regions. Since the performance of ET is closely related to the round-trip delay, a proxy-based ET can achieve better results, as reported in [2]. However, transmission errors are not corrected but only error propagation is prohibited, which reduces the perceived quality. On the other hand, the coding efficiency of ET is still limited, because a fixed part of the total bandwidth is reserved for error control. The need for error state handling and buffer space requirements are a further disadvantage common to reactive techniques.

Therefore, proactive techniques like FEC can provide better results in this particular scenario if applied properly. A straightforward Packet-level FEC (PEC) applied on the video server introduces constant overhead data which is undesirable for efficient data transmission. A better approach is to differentiate between more and less important data and apply the FEC accordingly, as it has been shown by unequal error protection schemes (UEP) in [3] and [4]. However, these approaches need intensive computations (decoding on packetlevel) which negatively affect the energy consumption of mobile devices. Furthermore, if applied end-to-end, they introduce unnecessary redundancy during the transmission in the wired part of the network.

Packet permutation is another proactive technique which makes video transmission more robust against bursts of errors. The idea is to randomize the sending packet order, which decreases the probability of losing important data during fading periods. Combined with PEC, packet permutation achieves a high recovery rate of QoS-essential video frames as reported in [5]. However, it also has the aforementioned drawbacks in terms of energy consumption.

In table 1, an overview about the characteristics of adaptive video transmission architectures is shown. We state that our RASMUS architecture provides better results as it achieves robustness by UEP application, coding efficiency by the combination of transcoding for the wired and Fine Granularity Scalability (FGS) together with UEP for the wireless part, and energy efficiency by avoiding large computations at the mobile client.

\begin{tabular}{l|l|l|l|l|l|c} 
& $\begin{array}{l}\text { Packet- } \\
\text { level } \\
\text { FEC } \\
\text { (PEC) }\end{array}$ & $\begin{array}{l}\text { EEP } \\
\text { UEP }\end{array}$ & $\begin{array}{l}\text { Error } \\
\text { Track- } \\
\text { ing } \\
\text { (ET) }\end{array}$ & $\begin{array}{l}\text { Proxy- } \\
\text { based } \\
\text { ET }\end{array}$ & $\begin{array}{l}\text { Packet } \\
\text { Per- } \\
\text { muta- } \\
\text { tion }+ \\
\text { PEC }\end{array}$ & RASMUS \\
\hline $\begin{array}{l}\text { Coding } \\
\text { Efficiency }\end{array}$ & - & 0 & 0 & 0 & - & + \\
\hline Robustness & o & o & o & + & + & + \\
\hline $\begin{array}{l}\text { Energy } \\
\text { Efficiency }\end{array}$ & - & - & + & + & - & +
\end{tabular}

Table 1. Adaptive Video Transmission Architectures

\section{SYSTEM ARCHITECTURE}

The goal of our two-level proxy architecture is to provide stream adaptation on the one hand as well as channel adaptation on the other hand. Therefore, a combination of transcoding techniques as well as the use of FGS and UEP is needed. Such a combination need neither be placed on one physical node nor in the same network. Thus, we propose to place a
Channel Adaptation Service Proxy (CAS Proxy) nearby the wireless channel and use a transcoding proxy from the Internet or from other service providers. From a qualitative perspective, the advantage of this approach is that less bandwidth will be consumed in the core network compared to approaches that apply end-to-end FEC or transcoding in the access network, while radio channel issues are handled as efficient as possible. In an UMTS network, the CAS Proxy can be placed at the RNC as illustrated in figure 1.

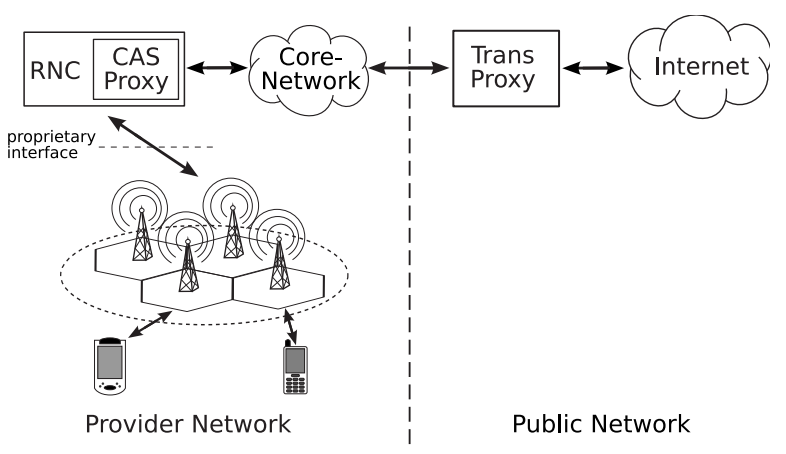

Fig. 1. System Architecture

The purpose of the transcoding proxy is to support heterogeneous mobile clients by providing for individual stream adaptation. For this, the coding format of the precoded multimedia data is changed through, e.g., temporal adaptation by reducing the frame rate, spatial adaptation by reducing the resolution of each frame, quality adaptation by reducing the number of bits used for coding each frame, or format adaptation by changing the coding format of the whole stream. A comprehensive overview of such architectures and transcoding techniques is given in [6]. Our approach is to combine those different transcoding techniques into a highly flexible transcoding proxy which can load several different transcoding modules at runtime according to the requirements of the client and the user. These client capabilities can be exchanged between proxy and client by the use of the optional RTSP methods GET/SET_PARAMETER.

\section{CHANNEL ADAPTATION}

We already mentioned that the wireless communication link has a highly dynamic behavior in terms of BER fluctuations and even bursts of errors. As it is stated in [7], it is more beneficial to map the varying channel conditions into a variable bit-rate channel. This can be achieved by the Channel Adaptive UEP in the proposed architecture (see figure 2). One important advantage of our proposed UEP scheme is that it adjusts the channel coding in the base station (Node-B) and therefore can benefit from efficient hardware implementation, which enables energy efficient data transmission over the wireless link. The adjustment settings include the type of channel coding (in UMTS: convolutional or turbo coding), code rate, i. e. the ratio between payload and the whole block size (UMTS supports two different code rates: $1 / 2$ and $1 / 3$ ), and the interleaver size, which denotes the amount of blocks to be treated by the physical layer (possible values in UMTS are 1,2, 4 and 8). Another advantage is that the UEP scheme not only considers the importance of the data, but also the current channel conditions (based on SNR measurements reports), which makes possible a near optimum allocation of redundancy on-the-fly. We assume that a dedicated channel 


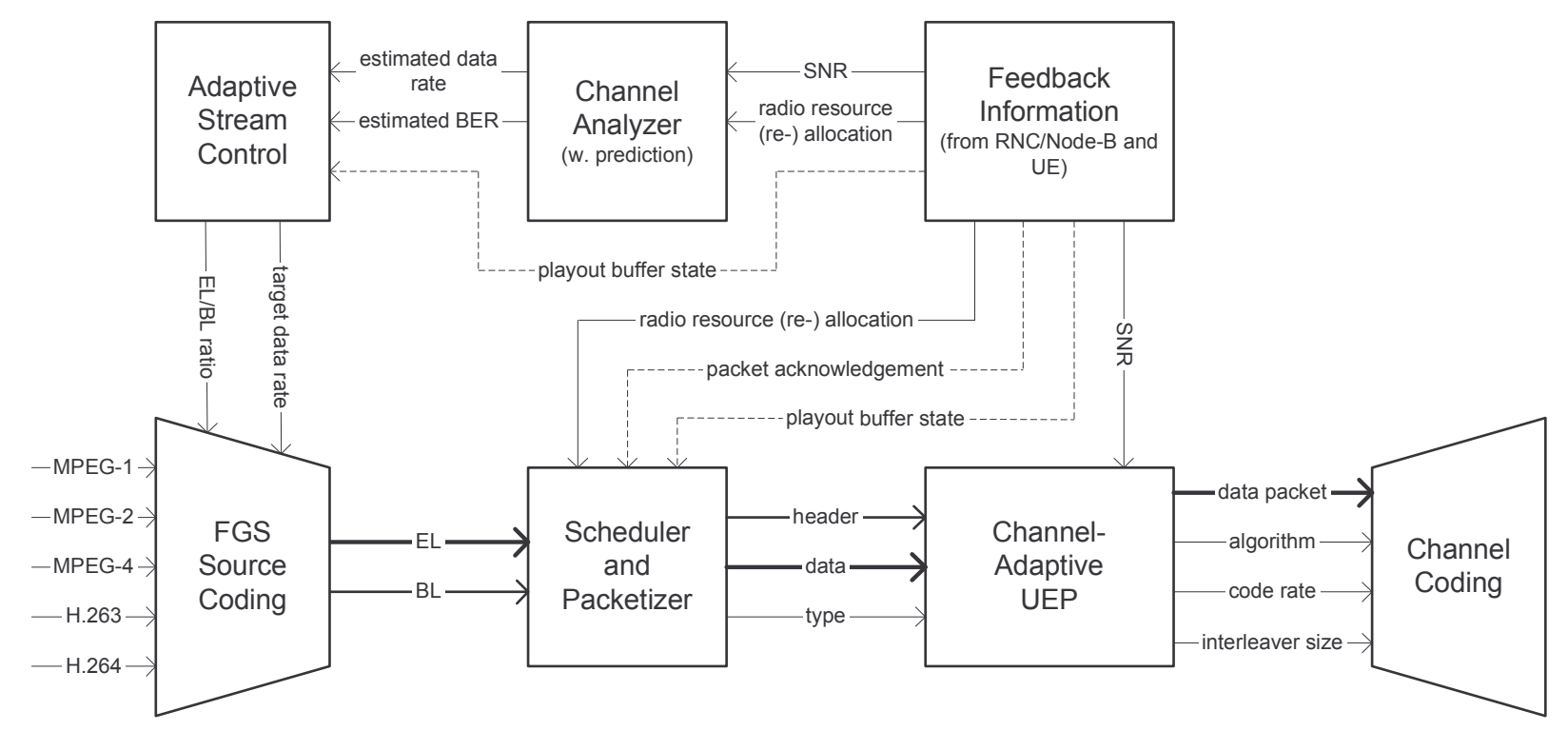

Fig. 2. Channel Adaptation Service Proxy

is established for the base layer data, while the enhancement layer can be transmitted over a shared channel when additional radio resources are available. Therefore, we expect a high error robustness and transmission efficiency over the wireless link.

In order to apply such an adaptive UEP scheme, the source coder should be able to provide an on-the-fly adjustment of the transmission bit-rate. Therefore, we have applied a FGS source coder which produces a video stream which is divided in two layers: the base layer which is a conventional (e.g. MPEG) video data with high quantization, and the enhancement layer which stores the differences between the base layer and the original content, and therefore improves the video quality [8]. It should be mentioned that every correctly received bit of the enhancement layer improves the perceptional quality. Since the source encoder can not adapt the target bitrate to the channel conditions immediately, prediction has to be in place in order to achieve optimal results. Therefore, we introduce the Channel Analyzer component which is responsible for proper prediction of the future radio channel conditions (available data rate and link quality in terms of BER) and triggers the Adaptive Stream Control (ASC) component. The ASC is responsible for the computation of the target bit-rate, as well as an appropriate ratio between base and enhancement layers based on the predicted channel conditions. Hence, a high perceptional video quality can be achieved.

Moreover, it should be mentioned that even though the CAS Proxy is able to operate without any feedback from higher layers of the UE, further improvements can be achieved when receiver reports are integrated (dashed lines in figure 2). For example, in case that a part of the base layer can not be decoded, the corrupted part may be retransmitted very fast because of the location of our CAS Proxy on the wireless interface. This will be done in the Scheduler and Packetizer component.

The transmission efficiency over the fixed wired part of the transmission path is ensured by optimal transcoding of the original video stream in the transcoding proxy.

\section{SIGNALING ASPECTS}

In a two-level proxy architecture, it is relevant how the transcoding and radio channel adaptation services can be composed. The requirements for this composition are, first, that it should be done in a user-friendly way and second, that the system components may be discovered and tied together according to their offered capabilities. The deployment of streaming services in 3G networks has been evaluated in [9], proposing a streaming architecture based on the IP Multimedia Subsystem (IMS) of UMTS. However, from a user perspective, it would be valuable to access any content in a consistent fashion. Besides, personalized services matching user preferences will gain more importance in future. We therefore focus on userfriendly service composition in our architecture, but mention that our architecture can be integrated with IMS to offer both IMS and external Internet-based services to users. We use a service composition approach similar to the one proposed in [10], but simplified to fit in our scenario. This approach supports a variety of other services in a mobile network and can be re-configured to support different QoS models or handover procedures.

We show an overview of our signaling architecture in fig. 3 . The User Equipment (UE) directs a request (step (1) in the figure) for the streaming service with the desired content and preferences regarding quality requirements to the Service Manager, whose address is pre-configured by the provider network. A further parameter of the streaming request is the location of the UE available from the VLR. The Service Manager will then (2) set up the Function Graph which in this case consists of Media Server (MS), Transcoding Proxy (TC) and Channel Adaptation Service Proxy (CASP). In order to actually establish a Service Path from MS to UE, an instance of each functional component must be located. In our case, it can be derived from the Function Graph that (a) the input format TC has to process depends on the output format of MS and the output format of the TC must be included in the user preferences, (b) CAS Proxy and MS do not depend directly, and (c) for each location of a UE at most one CAS Proxy can be located because of its location at the RNC. Thus, MS and CAS Proxy are located (3) in parallel before in step (4) the appropriate TC is located. In order to provide a notification for 
the user if the service is possible at all, the QoS on the resulting path should be checked (5). Since there may be several MS or TC, the best path may be selected according to load or network metrics.

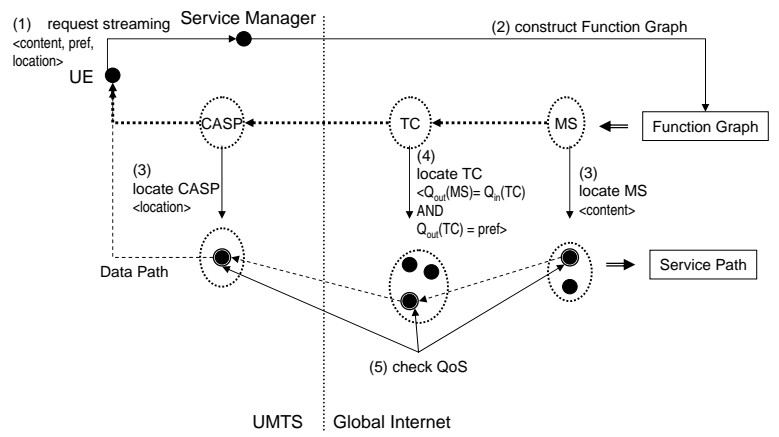

Fig. 3. Service Composition Process at Service Manager

The result of the service composition process at the Service Manager is a session configuration (i.e. URI of the content and Transcoding Proxy location) which will be sent to the User Equipment. The UE can open a usual streaming session then. Since the Transcoding Proxy is informed about the CAS Proxy address by the Service Manager, it can include it into the data path.

Depending on the underlying network, our signaling architecture may consider different QoS models. For example, in a future IMS based service, end-to-end QoS is guaranteed, whereas in today's Internet, QoS has not been widely deployed yet. Thus, the Service Manager will manage the available QoS requirements and parameters, and give notification on possible violation. Such QoS violations may occur at a handover of the mobile station. Using an IMS-based service, UMTS functionality can be applied to control QoS, but for an external IP service, QoS on the new path to a new CAS Proxy must be re-negotiated. Thus, we propose to examine the integration with Differentiated Services control plane approaches like [11].

\section{SUMMARY}

In this paper, we proposed a two-level proxy architecture to provide robust and adaptive video streaming. By applying a Transcoding Proxy which may reside near a content provider, both adaptation to user preferences and efficient bandwidth utilization in the core network is achieved. A Channel Adaptation Proxy nearby the radio channel offers both error resilience and coding efficiency with a combined FGS and UEP approach. Including feedback on channel state deals with the variations in radio channel characteristics. We have also mentioned service composition aspects to provide a user-friendly signaling of preferences, which is an important aspect for the success of IP-based multimedia services in future mobile networks.

Future work comprises the simulation and implementation of our architecture.

\section{REFERENCES}

[1] Bernd Girod and Niko Färber, "Feedback-Based Error Control for Mobile Video Transmission," Proceedings of The IEEE, Special Issue on Video for Mobile Multimedia, vol. 87, no. 10, pp. 1707-1723, October 1999.
[2] Wei Tu and Eckehard Steinbach, "Proxy-based error tracking for H.264 based real-time video transmission in mobile environments," in Proceedings of the IEEE International Conference on Multimedia and Expo (ICME'04), Taipei, Taiwan, June 2004.

[3] J. Cai, Qian Zhang, Wenwu Zhu, and C.W. Chen, "An FEC-based error control scheme for wireless MPEG4 video transmission," in Proceedings of the IEEE Wireless Communications and Networking Conference (WCNC'O0), Chicago, USA, September 2000.

[4] Qi Qu, Yong Pei, and James W. Modestino, "Robust H.264 video coding ans transmission over bursty packet loss wireless networks," in Proceedings of the 58th IEEE Vehicual Technology Conference (VTC'03), Orlando, USA, October 2003, pp. 3395-3399.

[5] Yueh-Min Huang, Shiang-Chun Liou, and Jen-Wen Ding, "An Novel Approach for Transmitting MPEG-4 Video over Burst-Error Wireless Channels," Multimedia Tools and Applications, vol. 23, no. 3, pp. 281-305, December 2003.

[6] Anthony Vetro, Charilaos Christopoulos, and Huifang Sun, "Video transcoding architectures and techniques: an overview," IEEE Signal Processing Magazine, vol. 20, no. 2, pp. 18-29, March 2003.

[7] Thomas Stockhammer, Jenkač Hrvoje, and Christian Weiß, "Feedback and Error Protection Strategies for Wireless Progressive Video Transmission," IEEE Transactions on Circuits and Systems for Video Technology, vol. 12, no. 6, pp. 465-482, June 2002.

[8] Weiping Li, "Overview of Fine Granularity Scalability in MPEG-4 Video Standard," IEEE Transactions on Circuits and Systems for Video Technology, vol. 11, no. 3, pp. 301-317, March 2001.

[9] Hector Montes, Gerardo Gomez, Renaud Cuny, and Jose F. Paris, "Deployment of IP Multimedia Streaming Services in Third-Generation Mobile Networks," IEEE Wireless Commmunications, vol. 9, no. 5, pp. 84 - 92, October 2002.

[10] Xiaohui Gu, Klara Nahrstedt, and Bin Yu, "SpiderNet: An Integrated Peer-to-Peer Service Composition Framework," in Proceedings of The IEEE International Symposium on High-Performance Distributed Computing (HPDC-13), Honolulu, Hawaii, June 2004.

[11] Joachim Hillebrand, Christian Prehofer, Roland Bless, and Martina Zitterbart, "Quality-of-Service Signaling for Next Generation IP-based Mobile Networks," IEEE Communications Magazine, vol. 42, no. 6, pp. 72-79, June 2004. 